\title{
Electrocardiographic analysis in a patient with dual atrioventricular nodal non-re-entrant tachycardia
}

\author{
Jing Chen ${ }^{1}$, Bei Wang ${ }^{2}$, Fan $\operatorname{Lin}^{2}$, Ping Zuo ${ }^{2}$, Li Lin², and Xiaoyun Yang ${ }^{2}$ \\ ${ }^{1}$ Tongji Hospital of Tongji Medical College of Huazhong University of Science and \\ Technology Department of Hematology \\ ${ }^{2}$ Tongji Hospital of Tongji Medical College of Huazhong University of Science and \\ Technology
}

April 28, 2020

\begin{abstract}
1:2 atrioventricular (AV) conduction is characterized by one atrial activation antegrade conduction down fast and slow pathways, which results in two ventricular activations. This phenomenon also referred to as a "double fire". When this phenomenon repeats rapidity, dual atrioventricular node non-re-entrant tachycardia (DAVNNT) occurs. We report the case of irregular QRS complexes tachycardia with a comprehensive record of this phenomenon including, routine electrocardiogram, Holter recordings, and intracardiac electrophysiological study (EPS). In conclusion, through analysis of the ECG and intracardiac recording during tachycardia, we presume that the most likely electrophysiological explanation of our case is the "double ventricular response to a single sinus beat".
\end{abstract}

\section{Hosted file}

Electrocardiographic analysis in a patient with dual atrioventricular nodal non-re-entrant tachycardia. available at https : //authorea.com/users/314194/articles/444543-electrocardiographic-analysisin-a-patient-with-dual-atrioventricular-nodal-non-re-entrant-tachycardia 